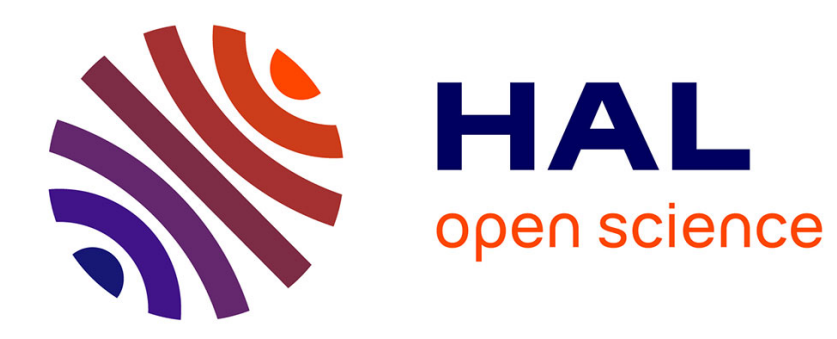

\title{
Convection driven by centrifugal buoyancy in nematics
}

\author{
C.R. Carrigan, E. Guyon
}

\section{To cite this version:}

C.R. Carrigan, E. Guyon. Convection driven by centrifugal buoyancy in nematics. Journal de Physique Lettres, 1975, 36 (5), pp.145-147. 10.1051/jphyslet:01975003605014500 . jpa-00231174

\section{HAL Id: jpa-00231174 https://hal.science/jpa-00231174}

Submitted on 1 Jan 1975

HAL is a multi-disciplinary open access archive for the deposit and dissemination of scientific research documents, whether they are published or not. The documents may come from teaching and research institutions in France or abroad, or from public or private research centers.
L'archive ouverte pluridisciplinaire HAL, est destinée au dépôt et à la diffusion de documents scientifiques de niveau recherche, publiés ou non, émanant des établissements d'enseignement et de recherche français ou étrangers, des laboratoires publics ou privés. 


\title{
CONVECTION DRIVEN BY CENTRIFUGAL BUOYANCY IN NEMATICS (*)
}

\author{
C. R. CARRIGAN
}

Department of Planetary and Space Science, University of California, Los Angeles, USA

and

\section{E. GUYON (**)}

Department of Physics, University of California, Los Angeles, USA

(Reçu le 6 février 1975, accepté le 27 février 1975)

\begin{abstract}
Résumé. - Un nématique contenu dans un anneau cylindrique vertical en rotation est chauffé par l'extérieur. Lorsque le directeur $\mathbf{n}$ est horizontal, les résultats confirment ceux obtenus en convection planaire usuelle. Une transition originale du $1^{\mathrm{er}}$ ordre, induite par les effets de force de Coriolis, est obtenue quand $\mathbf{n}$ est parallèle à l'axe du cylindre.
\end{abstract}

Abstract. - The onset of thermal convection in a nematic contained in a vertical rotating cylindrical annulus heated from the outside is studied as a function of the planar alignment of the liquid crystal director $\mathbf{n}$. When $\mathbf{n}$ is horizontal, the results confirm data on gravitational buoyancy. An original first order like convection mode is obtained when $\mathbf{n}$ is parallel to the cylinder axis.

Thermal convection in uniformly-aligned nematic liquid crystals is strongly modified both qualitatively and quantitatively with respect to the isotropic case [1] due to the coupling between the flow and molecular orientation [2]. In the planar configuration, considered here, the orientation characterized by a unit vector $\mathbf{n}$ - the director - and induced at the bounding surfaces is parallel to the plates. In gravity induced convection, the convective rolls are orientated perpendicular to $\mathbf{n}$ and the instability threshold is typically 500 times smaller than for an isotropic fluid of similar mean properties.

In this note, we study the case for which gravity $(g)$ is replaced by the centrifugal force $\left(\omega^{2} r\right)$ acting on a planar nematic contained between two concentric cylinders in solid body rotation about a vertical axis $\mathbf{z}$. The destabilizing temperature gradient is applied radially so that the outer temperature $T_{0}$ is larger than the inner one $T_{\mathrm{i}}, \Delta T=T_{0}-T_{\mathrm{i}}$. For isotropic fluids, it has been established both theoretically and experimentally [3] that the effect of centrifugal buoyancy is equivalent to that of gravitational buoyancy in the corresponding Rayleigh Benard problem $\left(g \leftrightarrow \omega^{2} r\right)$ when the effect of viscosity in the Ekman boundary layers can be neglected (the kine-

(*) Supported in part by NSF grants GA 31247 (C.R.C.) and GA 38867 (E.G.).

$\left(^{* *}\right)$ Also Laboratoire de Physique des Solides associé au C.N.R.S., 91405 Orsay, France. matic viscosity $v \approx 1$ cgs and the Ekman number $E=v / \omega r^{2}$ satisfies the inequality $E \ll(l / d)^{4}$; the height of the cell $l \sim 2.25 \mathrm{~cm}$, its width $d=750 \mu$ and the mean radius $r=2 \mathrm{~cm}$ ). However, the axes of the convection rolls have a preferred alignment along the axis of rotation when the Coriolis force is important. This alignment effect is a consequence of the Taylor-Proudman theorem. The theorem follows from the balance between the Coriolis force and the pressure gradient, in the hydrodynamic equations of motion. When this basic balance exists, the flow field must be independent of the coordinate along the axis of rotation. When the convection rolls are aligned along $\mathbf{z}$, the flow is essentially independent of $\mathbf{z}$. In this letter, we compare the aligning effects due to rotation and to the molecular orientation in the liquid crystalline state.

We use MBBA (methoxy p.n. benzilidene bubyl anilin) which is nematic at room temperature. The film is contained between two concentric lucite cylinders treated by polishing to provide the planar molecular alignment at the facing surfaces. The alignment in the upper half $(\mathrm{u})$ is horizontal (along the azimuthal $\theta$ axis, see Fig. 1). In the lower half (1), it is vertical (along $\mathbf{z}$ ). The temperature difference across the film, $\Delta T$, is obtained by circulating water at different and controlled temperatures on the outer walls of the annular cell. The $\Delta T$ values indicated here have been corrected to account for the finite thermal resistance of the cylinder walls. Stroboscopic and 


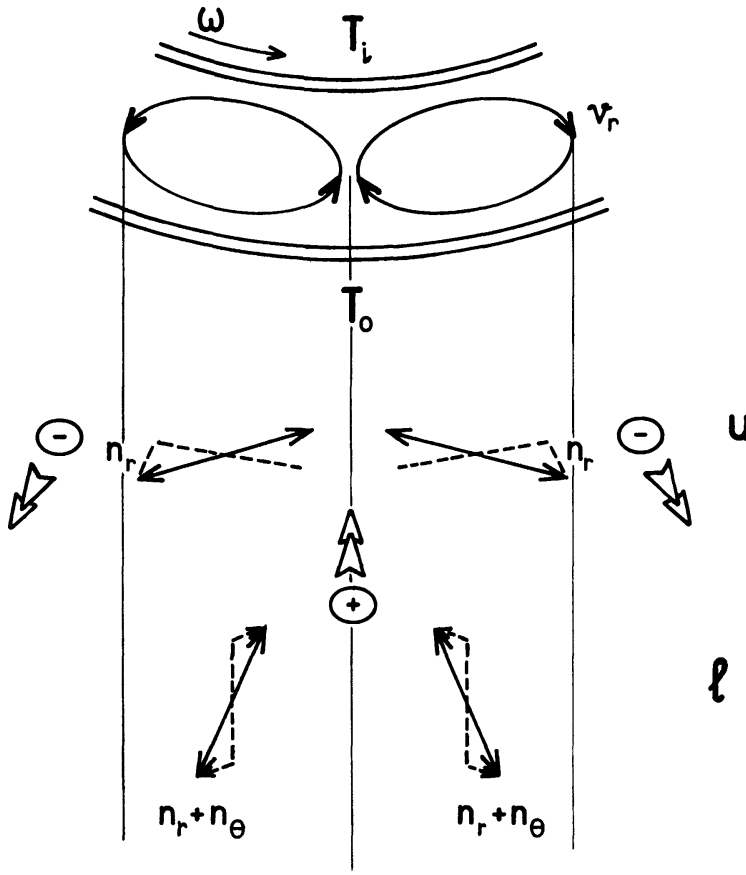

Schematic of the distortion of the nematic under the influence of the horizontal convective current.

Upper part the initial planar state $(\leftrightarrow \cdots)$ is distorted into $(\leftrightarrow)$. This takes place for infinitesimal velocity. Only the radial component $v_{\mathrm{r}}$ is considered in the one dimensional model.

Lower part above a critical value $v_{\mathrm{r}}$, the initial state is unstable with respect to the distorted ones.

In both cases, the distorsion focusses heat $(\oplus)$ in initially hotter regions. The instabilities involve vertical cylindrical rolls.

flash photographic observations of the cell rotating at angular velocities up to $\omega \sim 90 \mathrm{rps}$ are performed. A more detailed description of the apparatus is given in reference [3].

A typical experiment proceeds as follows : A given temperature difference is applied across the cell (e.g. $\Delta T=3^{\circ}$ ) and the rotation rate is increased progressively (between two successive observations we waited a standard time of $\mathbf{4 0}$ minutes in order to allow for the slow development of convection in nematics). In the upper half region, vertical convective rolls develop uniformly along the perimeter of the cylinder above a threshold value $\omega_{\mathrm{u}}(T)\left(\omega_{\mathrm{u}}\left(3^{\circ}\right)=43 \mathrm{rps}\right)$. The lower region remains undistorted up to much larger rotation rates where vertical convective rolls appear. However the roll structure is less uniform than in the upper half and regions with developed convection coexist with distorted ones. We characterise the rather loosely defined threshold by

$$
\omega_{1}^{\prime}(\Delta T)\left(\omega_{1}^{\prime}\left(3^{\circ}\right)=65 \mathrm{rps}\right) .
$$

When the rotation rate is decreased the rolls disappear uniformly in the upper part at the same value $\omega_{\mathrm{u}}(\Delta T)$. The threshold for complete disappearance of the lower rolls is much smaller $\left(\omega_{1}^{\prime \prime}\left(3^{\circ}\right)=35 \mathrm{rps}\right)$. The two different behaviors are indicative of a second order phase transition in the upper part and a first order one in the lower part.
Upper part. - In this case, both rotation effects and the horizontal molecular distortion favor vertical rolls. Experiments with different temperature gradients and a constant mean temperature indicate that $\Delta T$ varies a $\omega^{-2}$ as expected. The best fit gives

$$
\Delta T_{\text {cr }}=(0.55 \pm 0.15) 10^{4} / \omega^{2} r
$$

(cgs units). The value is in good agreement with the threshold for the gravitational instability, e.g., $\Delta T=7^{\circ}$ for the same thickness $d=750 \mu$. Let us note, as discussed in detail in [1], that the threshold is much smaller ( $\sim 500$ times) than the value predicted in an isotropic convection model : under the influence of convective currents, the molecular structure is distorted (Figure; $\mathrm{u})$; the radial distortion $n_{\mathrm{r}}(\ll 1)$ varies periodically along $\theta$. (In fact, the convective cells cannot be observed with ordinary light-polarized along $z$ ). In the MBBA liquid crystal phase, the heat conductivity parallel to the molecules is typically twice as large as that perpendicular [4]. In the presence of the distortion, heat is being focussed in the initially warmer region, which reinfores the instability. This is a powerful destabilizing mechanism because the relaxation time constant of the distortion is very long $\left(\sim 10^{4} \mathrm{~s}\right.$ for a $750 \mu$ film) [5].

Lower part. - The results obtained are more original. The existence of low thresholds $\omega_{1}$ and the change in the contrast of the roll structures when light polarization is rotated indicate that molecular distortion also takes place in the roll formation and acts to lower the threshold through the action of the anisotropic heat conduction.

In the undistorted state, the vertical director $\mathbf{n}$ is in a symmetric configuration with respect to horizontal fluctuating convective currents. However it has been shown [6] in shear flow experiments, with the initial condition that $\mathbf{n}$ was perpendicular to the velocity and velocity gradient, that such a state might be unstable with respect to a distorted one for large enough shears $s$. For MBBA, the threshold is given by

$$
s_{\mathrm{c}} d^{2}=2.4 \times 10^{-3} \mathrm{~mm}^{2} / \mathrm{s} \text {. }
$$

This corresponds to critical velocities

$$
v_{\mathrm{c}} \sim 1.5 \times 10^{-3} \mathrm{~mm} / \mathrm{s}
$$

in our case. Such an effect is consistent with the observation of a hysteretic phenomenon. The instability involving the periodic distortion of $\mathbf{n}$ off the vertical axis (Figure; 1) takes place only when the velocity fluctuation exceeds a critical value $v_{\mathrm{c}}$. When decreasing the rotation rate, the rolls are stable down to the value $\omega_{1}^{\prime \prime}(\Delta T)$ where the amplitude of the convecting currents is too small to sustain the molecular distortion. For a one-dimensional analysis, in which only the effect of the radial currents $v_{\mathrm{r}}(\theta)$ are considered, the distortion is such that the heat focussing effect is destabilizing as in the upper part (Figure; 1). In fact one should consider in detail the 
complete velocity field which has a rotational component as well as a straining one in order to obtain the sign of the distortion but the existence of a finite threshold is a general consequence of the initial symmetry and is independent of any detailed model. However an exact analysis of this finite amplitude convection is probably extremely delicate and beyond the descriptive intent of this work.

In conclusion, we have shown the influence of the Coriolis force on the value of convective thresholds for nematics. We have also demonstrated the existence of a first order phase transition (finite amplitude) convection [7] in experiments utilizing the centrifugal buoyancy force.

Acknowledgments. - We have had very useful discussions with Drs. F. H. Busse and P. Pieranski on this work. One of us (E. G.) wishes to thank the department of Physics at U.C.L.A. for the hospitality extended to him.

\section{References}

[1] Guyon, E. and Pieranski, P., C. R. Hebd. Séan. Acad. Sci. 274 (1972) 656.

Dubois-Violette, E., Pieranski, P. and Guyon, E., Mol. Cryst. \& Liq. Cryst. 26 (1974) 193.

[2] De Gennes, P. G., The Physics of Liquid Crystals (Oxford University Press) 1974.

[3] Busse, F. H. and Carrigan, C. R., J. Fluid Mech. 62 (1974) 579.

[4] Vilanove, R., Guyon, E., Mitescu, C. and Pieransky, P., J. Physique 35 (1974) 153.
[5] In a naive analysis, the convective threshold can be found to vary as the inverse of the relaxation time of the fluctuating variables (GuYon, E. and Pieranski, P., Physica 73 (1974) 184).

[6] Pieranski, P. and Guyon, E., Solid State Commun. 13(1973) 435.

[7] Similar effects have been obtained in electrohydrodynamic instabilities in nematics in the presence of a Poiseuille flow parallel to the director, GuYon, E. and PIERANSKI, P., to appear in C. R. Hebd. Séan. Acad. Sci. (January 1974). 